\title{
Optimised photometric stereo via non-convex variational minimisation
}

\author{
Laurent Hoeltgen ${ }^{1}$ \\ hoeltgen@b-tu.de \\ Yvain Quéau² \\ queau@enseeiht.fr \\ Michael Breuß ${ }^{1}$ \\ breuss@b-tu.de \\ Georg Radow ${ }^{1}$ \\ radow@b-tu.de
}

\author{
${ }^{1}$ Chair for Applied Mathematics \\ BTU Cottbus-Senftenberg, \\ Cottbus, Germany \\ ${ }^{2}$ IRIT, UMR CNRS 5505 \\ Université de Toulouse \\ Toulouse, France
}

Estimating the shape and appearance of a three dimensional object from flat images is a challenging research topic that is still actively pursued. Among the various techniques available, Photometric Stereo (PS) is known to provide very accurate local shape recovery in terms of surface normals. In this work we propose to minimise non-convex variational models for PS that recover the depth information directly.

Photometric Stereo consists in finding a depth map $z$ that best explains all image irradiance equations (IIEs) $I^{i}=\mathcal{R}\left(z ; s^{i}, \rho\right)$, for several images $I^{i}$, considered under different lightings $s^{i}$, with $i \in\{1, \ldots, m\}$. The function $\mathcal{R}$ describes our reflectance model in terms of the depth $z$, the lighting $s^{i}$, and the albedo $\rho$. We assume Lambertian reflectance, neglect shadows, and require $m \geqslant 3$.

Our approach uses a variational framework with a least-squares penalisation on the IIEs augmented with a zero-th order Tikhonov regularisation. The obtained energy (1) is non-convex and we make use of matrix differential theory and recent developments in non-convex and nonsmooth optimisation to determine good minimisers.

$$
\min _{z, \rho}\left\{\mathcal{E}_{\mathcal{R}}(z, \rho ; I)+\frac{\lambda}{2}\left\|z-z_{0}\right\|^{2}\right\}
$$

Here, $\mathcal{E}_{\mathcal{R}}$ represents the reprojection criterion based on the IIEs.

Our numerical strategy uses recent findings of Ochs et al. [2]. They proposed a novel method to handle such tasks, called iPiano. Inspired by the heavy-ball method, it separates non-smooth and non-convex parts in an efficient splitting strategy. Further benefits include a thorough convergence theory. The algorithm makes explicit use of the derivative of the smooth terms in the cost function, which in our case involves derivatives of matrix-valued functions, and we will employ
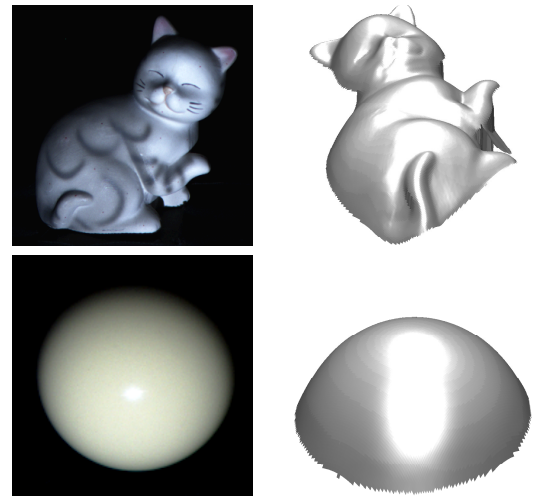

Figure 1: Test data, 3D-reconstructions obtained, 3D-reconstruction results using the full pipeline, consisting of a preprocessing, followed by classic PS and finally our proposed method.

as a technical novelty, matrix differential theory [1] to derive the resulting scheme.

Figure 1 presents some visualised results. It consists of two scenes captured under 20 different known illuminants. The experimental setups also demonstrate that our framework performs consistently better than alternative approaches such as [3] in terms of the mean angular error.

[1] J. R. Magnus and H. Neudecker. Matrix Differential Calculus with Applications in Statistics and Econometrics. John Wiley \& Sons, 3rd edition, 2007.

[2] P. Ochs, Y. Chen, T. Brox, and T. Pock. iPiano: Inertial proximal algorithm for non-convex optimization. SIAM Journal on Imaging Sciences, 7 (2):1388-1419, 2014.

[3] R. J. Woodham. Photometric method for determining surface orientation from multiple images. Optical Engineering, 19(1):134-144, 1980. 